\title{
Análise de Sensibilidade de Primeira Ordem Aplicada a Sistemas Elétricos de Potência
}

\author{
William M. da Rosa ${ }^{1}$, Priscila Rossoni ${ }^{2}$, Edmarcio A. Belati $^{3}$, Edmea C. Baptista $^{4}$ \\ $(1,2,3)$ - Centro de Engenharia, Modelagem e Ciências Sociais Aplicadas (CECS) \\ UFABC - Santa Adélia, 166 - Bairro Bangu - Santo André /SP - Brasil - CEP 09.210-170 \\ e-mal: ${ }^{1}$ william.moreti@ufabc.edu.br, ${ }^{2}$ priscila.rossoni@ ufabc.edu.br, ${ }^{3}$ edmarcio.belati@ufbac.edu.br \\ (4) Departamento de Matemática \\ FC - UNESP - Campus de Bauru - Av. Luis E. C. Coube, S/N - Bauru/ SP - Brasil CEP 17033-360 \\ e-mail: baptista@fc.unesp.br
}

Resumo: Neste trabalho é apresentado um estudo em sistemas de distribuição de energia elétrica utilizando uma técnica de análise de sensibilidade de primeira ordem (AS), que é aplicada à solução do algoritmo de Fluxo de Carga (FC). Diferente desse algoritmo, o método de análise de sensibilidade não requer um processo iterativo, resultando em uma metodologia rápida com grande precisão. Iniciando de uma solução conhecida do Fluxo de Carga considerada como caso base, novos pontos de operação podem ser calculados de forma direta depois de realizadas perturbações nas cargas do sistema. A técnica de análise de sensibilidade foi aplicada nos sistemas de transmissão de 14 barras e distribuição de 70 barras. Os resultados demonstram a eficiência da metodologia.

\section{INTRODUÇÃO}

Os sistemas elétricos de potência (SEP's) vêm operando cada vez mais perto dos limites máximos de carregamento e essa situação deve continuar nos próximos anos. O crescimento da demanda acompanhado de restrições econômicas e ambientais tem levado os SEP's a operar cada vez mais perto de seus limites operacionais. O consumo de energia elétrica mundial aumentará em $84 \%$ no período correspondente entre 2008 e 2035 [8].

A AS é de grande importância nos estudos da operação dos SEP's, pois ela ajuda no entendimento da relação causa-efeito entre os parâmetros do sistema e pode ser usada em algumas aplicações na operação em tempo real, devido à obtenção de uma relação direta entre as variáveis de controle e controladas, aspecto importante no contexto de Smart Grids [9]. Para tanto, se torna necessário o desenvolvimento de novas metodologias e a avaliação das existentes, de modo a possibilitar uma análise mais segura e eficiente dos SEP's em virtude das variações de seus parâmetros.

Em programação matemática, técnicas de AS [4, 5, 6, 10, 11, 13] têm sido usadas para obter condições de otimalidade, resultados duais, solução de algoritmos, taxa de convergência e aceleração de convergência de algoritmos, em adição a suas mais óbvias e imediatas aplicações em estimar soluções mais próximas com diferentes parâmetros.

O problema deste trabalho consiste em aplicar uma perturbação no ponto de operação obtido na solução do FC utilizando o modelo matemático descrito por [1, 2, 7, 12, 14,15], considerado como a solução para o caso base, e através de análise de sensibilidade estimar o novo ponto de operação. Assim, será possível calcular a sensibilidade de uma variável elétrica como a tensão $V_{i}$ no nó i em relação à injeção de potência ativa $P_{j}$ no nó $\mathrm{j}$ e projetar o comportamento de uma variável em relação às alterações nas injeções de potência.

\section{FLUXO DE CARGA}

O cálculo do FC em um sistema de energia elétrica consiste na obtenção do estado do sistema para uma dada condição de carga, geração e topologia de rede. A formulação básica do problema pode ser encontrada em MONTICELLI [12], onde a cada barra do sistema associam-se quatro variáveis:

$V_{k}$ - magnitude de tensão na barra $k$;

$\theta_{k}$ - ângulo de fase da barra $k$; 
$P_{k}$ - injeção de potência ativa na barra $k$;

$Q_{k}$ - injeção de potência reativa na barra $k$.

\section{Expressões Gerais dos Fluxos}

As injeções de potência ativa e reativa são obtidas impondo-se a Lei de Kirchhoff das correntes em cada barra, podendo ser calculadas na forma polar, respectivamente, com a Eq. (1) e Eq. (2).

$$
\begin{aligned}
& \mathrm{P}_{\mathrm{k}}(\mathrm{V}, \theta)=\mathrm{V}_{\mathrm{k}} \sum_{\mathrm{m} \in \mathrm{k}} \mathrm{V}_{\mathrm{m}}\left(\mathrm{G}_{\mathrm{km}} \cos \theta_{\mathrm{km}}+\mathrm{B}_{\mathrm{km}} \operatorname{sen} \theta_{\mathrm{km}}\right) \\
& \mathrm{Q}_{\mathrm{k}}(\mathrm{V}, \theta)=\mathrm{V}_{\mathrm{k}} \sum_{\mathrm{m} \in \mathrm{k}} \mathrm{V}_{\mathrm{m}}\left(\mathrm{G}_{\mathrm{km}} \operatorname{sen} \theta_{\mathrm{km}}-\mathrm{B}_{\mathrm{km}} \cos \theta_{\mathrm{km}}\right)
\end{aligned}
$$

Em que:

$G_{k m}$ - elemento real da matriz $\boldsymbol{Y}_{B A R R A}$ relacionado com as barras $k$ e $m$;

$B_{k m}$ - elemento imaginário da matriz $\boldsymbol{Y}_{B A R R A}$ relacionado com as barras $k$ e $m$;

$m \in k$ - conjunto de todas as barras $m$ que possuem ligação com a barra $k$.

A solução do problema de FC consiste em resolver as equações de balanço de potência ativa e reativa dadas respectivamente pelas Eq. (3) e Eq. (4).

$$
\begin{aligned}
& \Delta \mathrm{P}_{\mathrm{k}}=\mathrm{P}_{\mathrm{k}}^{\mathrm{esp}}-\mathrm{P}_{\mathrm{k}}^{\text {calc }}(\boldsymbol{V}, \boldsymbol{\theta})=0 \\
& \Delta \mathrm{Q}_{\mathrm{k}}=\mathrm{Q}_{\mathrm{k}}^{\text {esp }}-\mathrm{Q}_{\mathrm{k}}^{\text {calc }}(\boldsymbol{V}, \boldsymbol{\theta})=0
\end{aligned}
$$

O sobrescrito $\boldsymbol{e s p}$ representa os valores especificados de injeção de potência nas barras que são considerados constantes (modelo de carga de potência constante). O sobrescrito calc representa os valores calculados das injeções das potências obtidos a partir dos vetores das variáveis de estado $(\boldsymbol{V}, \boldsymbol{\theta})$ e dos parâmetros do sistema.

\section{TÉCNICA DE SENSIBILIDADE}

Como descrito a AS é de grande importância nos estudos de operação dos SEP's. Ela auxilia no entendimento da relação causa-efeito existente entre os parâmetros do sistema e pode ser usada em aplicações na operação em tempo real.

Vamos considerar dois tipos de variáveis: as variáveis operacionais denotadas pelo vetor $\boldsymbol{u}$; e as variáveis controladas denotadas pelo vetor $\boldsymbol{x}$. Nesse estudo, temos:

$\boldsymbol{x}$ - vetor de variáveis de estado do problema $(\boldsymbol{V}, \boldsymbol{\theta})$;

$\boldsymbol{u}$ - vetor de injeção das potências ativas e reativas nas barras $\left(\boldsymbol{P}_{\boldsymbol{i n j}}, \boldsymbol{Q}_{\boldsymbol{i n j}}\right)$.

As equações de fluxo de potência ativa e reativa, Eq. (3) e Eq. (4), podem ser expressas em notação vetorial como sendo:

$$
\mathrm{g}(\mathrm{x}, \mathrm{u})=0
$$

Supondo que $\boldsymbol{x}=\boldsymbol{x}^{*}$ é a solução para o vetor de controle especificado $\boldsymbol{u}=\boldsymbol{u}^{*}$ que satisfaz a Eq. (5), então:

$$
\mathrm{g}\left(\mathrm{x}^{*}, \mathrm{u}^{*}\right)=0
$$

Sabendo que uma mudança $\Delta \boldsymbol{u}$ em $\boldsymbol{u}^{*}$, causa uma mudança $\Delta \boldsymbol{x}$ de $\boldsymbol{x}^{*}$, aplica-se a expansão da série de Taylor na Eq. (6), obtendo-se:

$$
\mathrm{g}\left(\mathrm{x}^{*}+\Delta \mathrm{x}, \mathrm{u}^{*}+\Delta \mathrm{u}\right)=\mathrm{g}\left(\mathrm{x}^{*}, \mathrm{u}^{*}\right)+\mathrm{S}_{\mathrm{x}} \Delta \mathrm{x}+\mathrm{S}_{\mathrm{u}} \Delta \mathrm{u}=0
$$

A matriz $\boldsymbol{S}_{\boldsymbol{x}}$ é idêntica a matriz Jacobiana, sendo assim ela pode ser escrita da seguinte forma:

$$
\mathrm{S}_{\mathrm{X}}=\mathrm{J}=\left[\begin{array}{ll}
\frac{\partial \Delta \mathrm{P}}{\partial \mathrm{V}} & \frac{\partial \Delta \mathrm{P}}{\partial \theta} \\
\frac{\partial \Delta \mathrm{Q}}{\partial \mathrm{V}} & \frac{\partial \Delta \mathrm{Q}}{\partial \theta}
\end{array}\right]
$$


A matriz $\boldsymbol{S}_{\boldsymbol{u}}$ é obtida como segue:

$$
\mathrm{S}_{\mathrm{u}}=\left[\begin{array}{ll}
\frac{\partial \Delta \mathrm{P}}{\partial \mathrm{P}} & \frac{\partial \Delta \mathrm{P}}{\partial \mathrm{Q}} \\
\frac{\partial \Delta \mathrm{Q}}{\partial \mathrm{P}} & \frac{\partial \Delta \mathrm{Q}}{\partial \mathrm{Q}}
\end{array}\right]
$$

A solução da matriz $\boldsymbol{S}_{u}$ resulta em uma matriz identidade, quando for considerado o modelo de injeções de potência constante.

Combinando-se as equações (6) e (7) tem-se:

$$
\mathrm{S}_{\mathrm{x}} \Delta \mathrm{x}+\mathrm{S}_{\mathrm{u}} \Delta \mathrm{u}=0
$$

Reorganizando tem-se:

$$
\Delta \mathrm{x}=-\mathrm{S}_{\mathrm{x}}^{-1} \mathrm{~S}_{\mathrm{u}} \Delta \mathrm{u}=0
$$

Para simplificar a expressão (11):

$$
\mathrm{S}=-\mathrm{S}_{\mathrm{x}}^{-1} \mathrm{~S}_{\mathrm{u}}
$$

A $\boldsymbol{S}_{u}$ sendo uma matriz identidade a $\boldsymbol{S}$ fica idêntica a $\boldsymbol{J}^{\mathbf{- 1}}$, utilizada na última iteração do FC Assim, temos a expressão para correção do vetor $\Delta \boldsymbol{x}$.

$$
\Delta \mathrm{x}=\mathrm{J}^{-1} \Delta \mathrm{u}
$$

A Eq. (13) pode ser escrita na forma matricial como (14), em que NPQ é o número de barras de carga do sistema de distribuição:

$$
\left[\begin{array}{c}
\Delta \mathrm{x}_{1} \\
\Delta \mathrm{x}_{2} \\
\vdots \\
\Delta \mathrm{x}_{(2 \mathrm{NPQ})}
\end{array}\right]=\left[\begin{array}{ll}
\frac{\partial \Delta \mathrm{P}}{\partial \theta} & \frac{\partial \Delta \mathrm{P}}{\partial \mathrm{V}} \\
\frac{\partial \Delta \mathrm{Q}}{\partial \theta} & \frac{\partial \Delta \mathrm{Q}}{\partial \mathrm{V}}
\end{array}\right]^{-1}\left[\begin{array}{c}
\Delta \mathrm{u}_{1} \\
\Delta \mathrm{u}_{2} \\
\vdots \\
\Delta \mathrm{u}_{(2 \mathrm{NPQ})}
\end{array}\right]
$$

O equacionamento apresentado foi desenvolvido para sistemas de distribuição com uma barra sendo a subestação e as demais barras de carga.

Como mencionado, o vetor $\boldsymbol{u}$ consiste de variáveis independentes que são as injeções de potência ativa e reativa nas barras. O vetor $\boldsymbol{x}$ consiste de variáveis controladas que são as magnitudes das tensões e os ângulos de fase nas barras. Desta forma, o sistema matricial (14) pode ser reescrito como:

$$
\left[\begin{array}{c}
\Delta \theta_{1} \\
\Delta \theta_{2} \\
\vdots \\
\Delta \theta_{(\mathrm{NPQ})} \\
\Delta \mathrm{V}_{1} \\
\Delta \mathrm{V}_{2} \\
\vdots \\
\Delta \mathrm{V}_{(\mathrm{NPQ})}
\end{array}\right]=[\mathrm{J}]^{-1}\left[\begin{array}{c}
\mathrm{P}_{\mathrm{inj}_{1}} \\
\mathrm{P}_{\mathrm{inj}_{1}} \\
\vdots \\
\mathrm{P}_{\mathrm{inj}_{(\mathrm{NPQ})}} \\
\mathrm{Q}_{\mathrm{inj}_{1}} \\
\mathrm{Q}_{\mathrm{inj}_{1}} \\
\vdots \\
\mathrm{Q}_{\mathrm{inj}_{(\mathrm{NP})}}
\end{array}\right]
$$

O sistema matricial, dado por (15) é composto pelo vetor perturbação no lado direito do sistema matricial, em que são consideradas as perturbações nas injeções de potência ativa e reativa; a inversa da matriz $J$; e o vetor de correção das variáveis de estado no lado esquerdo da igualdade.

Com o sistema matricial, Eq. (14), novas soluções podem ser obtidas quando perturbações são realizadas nas injeções de potência nas barras através do vetor $\boldsymbol{x}_{n e w}$, Eq. (16).

$$
\mathrm{x}_{\text {new }}=\Delta \mathrm{x}+\mathrm{x}^{*}
$$




\section{Passos do Algoritmo}

Os passos para obtenção do $\boldsymbol{x}_{\text {new }}$ com a técnica de AS apresentada são os seguintes:

i. Entrar com os dados do sistema;

ii. Obter o estado do sistema via Fluxo de Carga;

iii. Entrar com o vetor perturbação, $\Delta \mathrm{u}$;

iv. Utilizar a Eq. (14) para calcular $\Delta x$;

v. Utilizar a Eq. (16) para obter o vetor $\mathrm{x}_{\text {new }}$;

vi. Se desejar realizar uma nova perturbação voltar ao passo (iii); Caso contrário - Fim.

Na aplicação da técnica de análise de sensibilidade, é resolvido apenas um FC, cuja solução é armazenada juntamente com os valores da Matriz Jacobiana, obtida na solução do fluxo de carga (caso base). Assim, o processo é direto na obtenção da solução, despendendo pouco recurso computacional.

\section{RESULTADOS E TESTES COMPUTACIONAIS}

Nesta seção será apresentado um teste com um sistema didático e testes com o sistema de transmissão de 14 barras [16] e distribuição de 70 barras [3].

\section{Teste Matemático}

Seja o seguinte problema:

$$
\begin{gathered}
x_{1}+2 x_{2}-1=\epsilon \\
x_{1}{ }^{2}-x_{2}=0
\end{gathered}
$$

Resolvendo o problema para $\epsilon=0$ temos: $x_{1}=0,5$ e $x_{1}=0,25$, conforme a Tabela 1 . Essa solução é considerada a solução para o caso base.

Tabela.1: Solução para o problema 17

\begin{tabular}{|c|c|c|}
\hline$\epsilon=0$ & $x_{1}$ & $x_{2}$ \\
\hline$'+'$ & 0.5000 & 0.2500 \\
\hline
\end{tabular}

Utilizando a técnica de análise de sensibilidade, foi realizada uma perturbação de $\epsilon=0,1 \mathrm{e}$ $\epsilon=0,2$ e o resultado obtido comparado com a solução exata do problema. A Figura 1 mostra essa comparação.

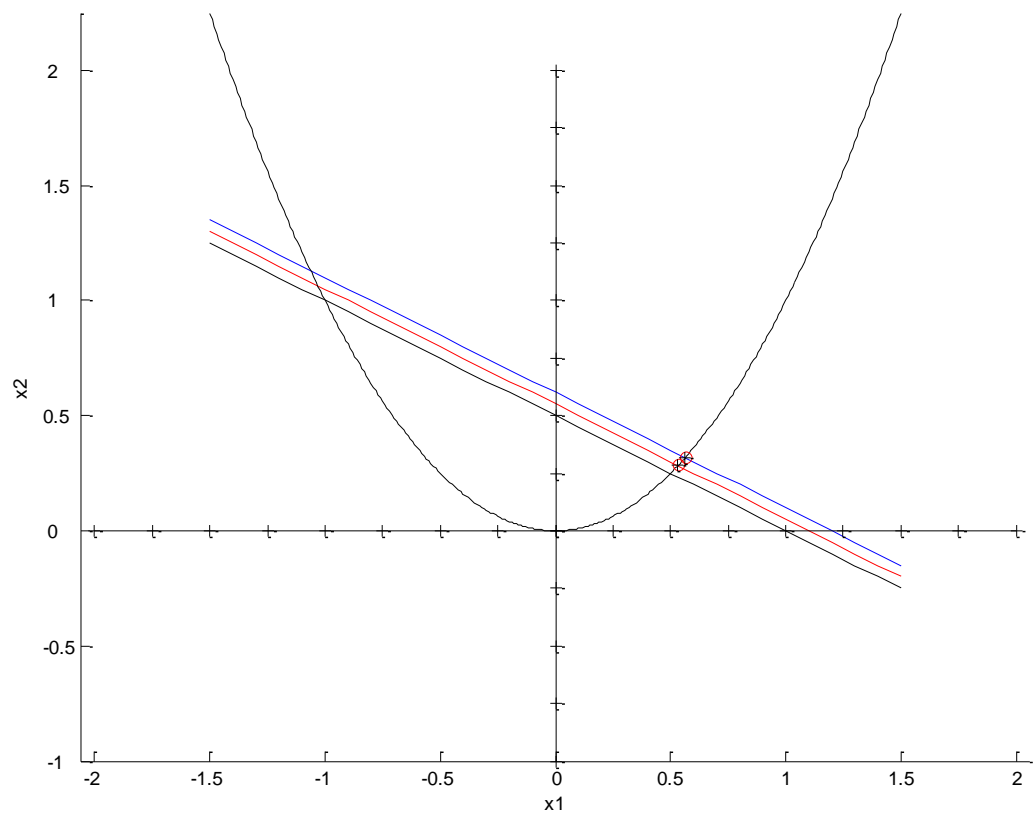

Figura 1: Solução com perturbações de $\epsilon=0,1$ e $\epsilon=0,2$ para o problema (17) 
A reta em preto representa a solução para o caso base e as retas em vermelho e azul a solução para $\epsilon=0,1$ e $\epsilon=0,2$ respectivamente. O circulo (O) em vermelho representa o ponto estimado e o mais (+) o ponto calculado. A Tabela 2 apresenta os valores obtidos e a Figura 2 um "zoom" da solução.

Tabela.2: Valores obtidos para as perturbações

\begin{tabular}{|c|c|c|c|c|}
\hline & $x_{1}$ & $x_{2}$ & $x_{1}$ & $x_{2}$ \\
\hline & \multicolumn{3}{|c|}{ Calculado ‘+' } & \multicolumn{2}{c|}{ Estimado via $\mathrm{AS}^{`} \mathrm{O}^{\prime}$} \\
\hline$\epsilon=0,1$ & 0,5326 & 0,2836 & 0,5333 & 0,2833 \\
\hline$\epsilon=0,2$ & 0,5639 & 0,3180 & 0,5666 & 0,3166 \\
\hline
\end{tabular}

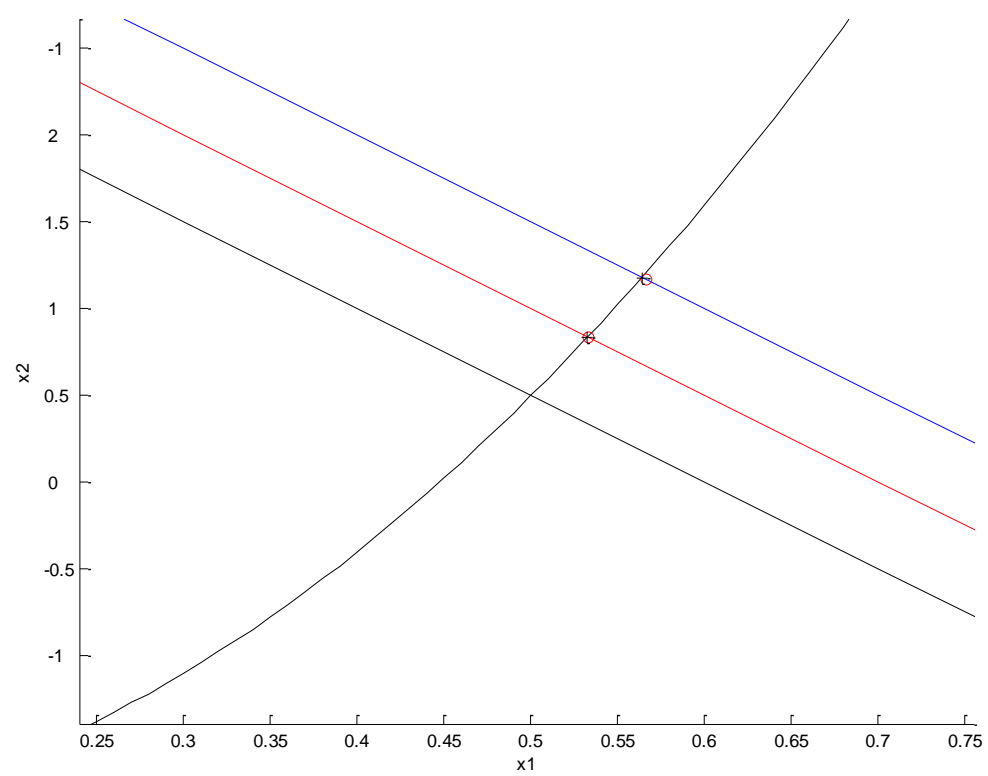

Figura 2: "Zoom" da solução com perturbações de $\epsilon=0,1$ e $\epsilon=0,2$ para o problema (17)

O exemplo mostra que a técnica de AS pode ser aplicada em sistemas não lineares. O ponto estimado ficou próximo do ponto calculado, o que viabiliza sua aplicação em problemas com variações nas restrições de igualdade.

\section{Testes para os sistemas de Transmissão e Distribuição de Energia Elétrica}

Neste teste foram utilizados os sistemas de transmissão de 14 barras e o sistema de distribuição de 70 barras para ilustrar a aplicabilidade da metodologia em redes de transmissão e distribuição de energia elétrica. Os dados dos sistemas podem ser obtidos em [3,16].

Para os sistemas citados acima foram realizados estudos do comportamento das perdas ativas em sistemas de transmissão e distribuição. Iniciando com o caso base, as potências ativas e a reativas nas barras de carga foram aumentadas de 2, 4, 6\%, até o valor máximo de 50\%, que pode ser considerado uma grande perturbação. Para cada $2 \%$ de perturbação, a técnica de AS destacada como "Sens" foi aplicada e o novo estado do sistema estimado. As Figuras 3 e 4 mostram os resultados obtidos comparados com a solução exata utilizando o FC. O resultado mostra que a técnica de AS pode ser utilizada para estimar as perdas.

Tabela.3: Comparação Do Tempo Computacional De Processamento

\begin{tabular}{|c|c|c|c|}
\hline Sistema Elétrico & Tempo (ms) - FC & Tempo (ms) - Sens & Ganho (\%) Sens/FC \\
\hline Sistema de 14 Barras & 27,1 & 0,07940 & 99,71 \\
\hline Sistema de 70 Barras & 6,8 & 1,8 & 99,73 \\
\hline
\end{tabular}

Vale destacar que, no código desenvolvido para ambiente MATLAB, o tempo gasto para estimar a solução via AS foi aproximadamente $99 \%$ mais rápida que utilizando o FC resolvido através do 
método de NR. Os tempos aproximados para os sistemas de 14 e 70 barras, podem ser verificados na Tabela 3.

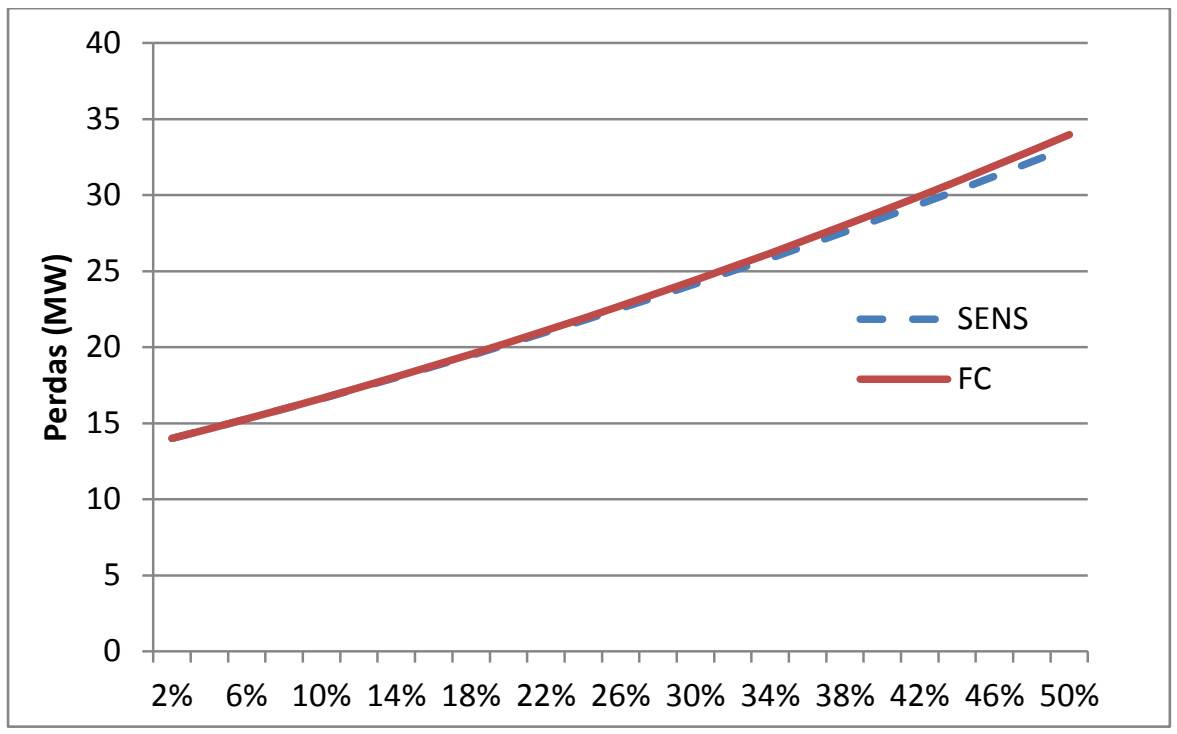

Figura 3: Comparação das perdas no sistema de 14 barras

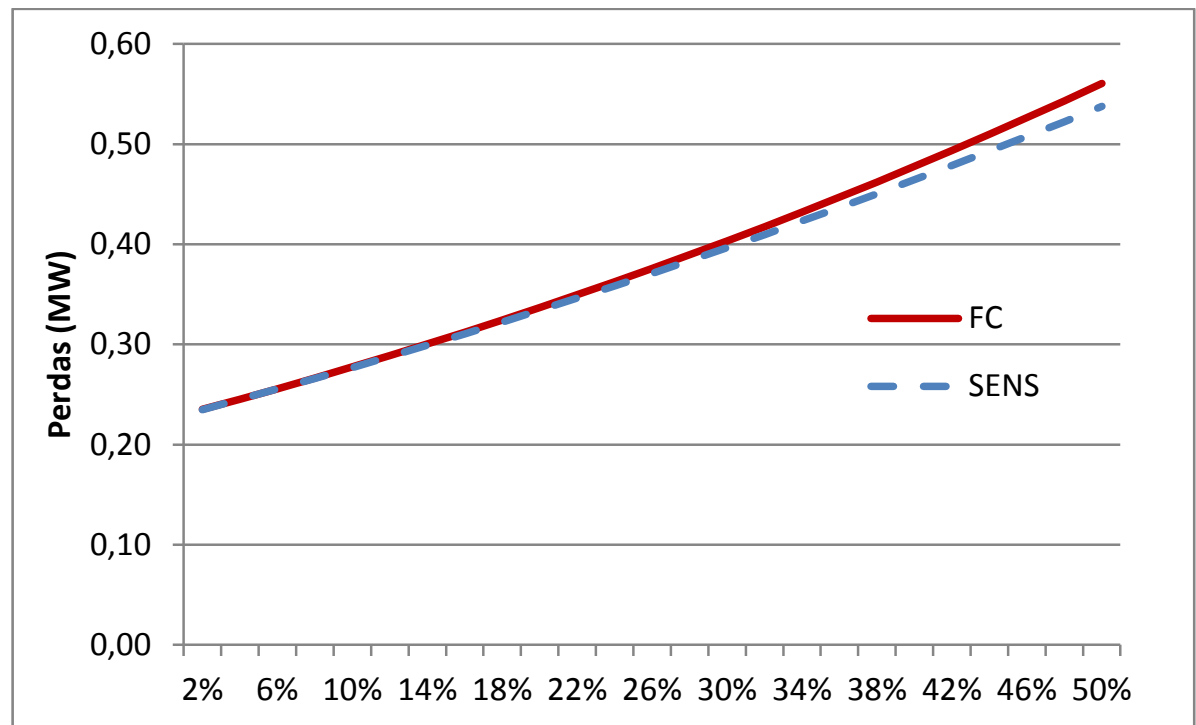

Figura 4: Comparação das perdas no sistema de 70 barras

Os maiores erros nas potências ativas e reativas, Eq. (3) e Eq. (4) respectivamente, aconteceram na barra 66. A Figura 5 apresenta o $\Delta \mathrm{P}_{66}$ e a Figura $6 \Delta Q_{66}$.

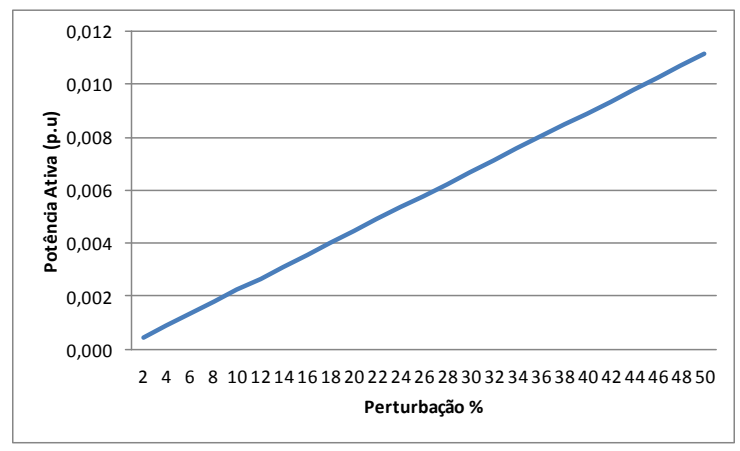

Figura 5: Erro da potência ativa na barra 66

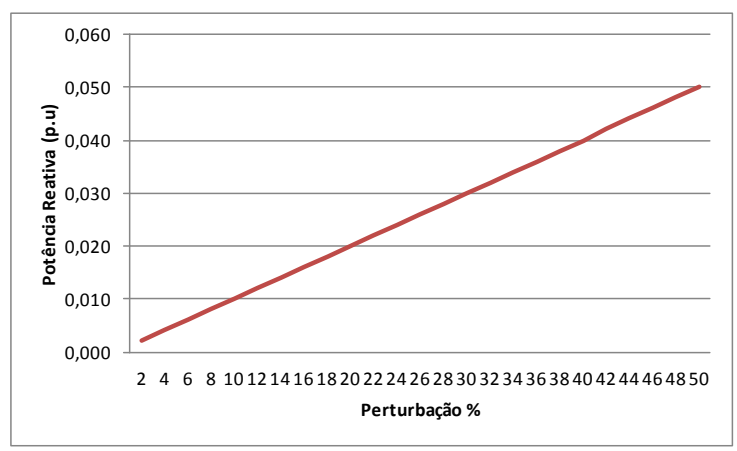

Figura 6: Erro da potência reativa na barra 66

Os gráficos dos erros mostram que a aplicação de AS pode ser utilizada na operação de redes de distribuição. 


\section{CONCLUSÕES:}

O FC é uma importante ferramenta utilizada no planejamento e operação de SEP's. O objetivo da aplicação do FC é obter os ângulos e as magnitudes de tensão para as barras da rede. Este artigo relata um estudo sobre o funcionamento de um SEP com AS. A metodologia utilizada consiste em aplicar uma perturbação no ponto de operação obtido na solução de do FC, considerado como a solução para o caso base, e através de AS estimar o novo ponto de operação. Assim, foi possível calcular a sensibilidade de uma variável elétrica como relação à outra. No estudo, perturbações foram introduzidas nas barras de carga até $50 \%$ dos sistemas de transmissão 14 barras e de distribuição de 70 barras. Observou-se que as perdas de potência ativa fornecida pela AS e PC são muito semelhantes. A principal vantagem da abordagem de AS é que, ao contrário do FC, não é iterativo e tem baixo custo computacional.

\section{AGRADECIMENTOS}

Os autores agradecem a Coordenação de Aperfeiçoamento de Pessoal de Nível Superior (CAPES) pela bolsa de estudo de William M. da Rosa.

\section{REFERÊNCIAS BIBLIOGRÁFICAS}

[1] T. L. Baldwin and S. A. Lewis, "Distribution load flow methods for shipboard power systems," Industry Applications, IEEE Transactions on, vol. 40, pp. 1183-1190, 2004.

[2] M. E. Baran and F. F. Wu, "Network reconFiguration in distribution systems for loss reduction and load balancing," Power Delivery, IEEE Transactions on, vol. 4, pp. 1401-1407, 1989.

[3] M. E. Baran and F. F. Wu, "Optimal capacitor placement on radial distribution systems," Power Delivery, IEEE Transactions on, vol. 4, pp. 725-734, 1989.

[4] E. A. Belati, E. C. Baptista, and G. R. M. da Costa, "Optimal operation studies of the power system via sensitivity analysis," Electric Power Systems Research, vol. 75, pp. 79-84, 7// 2005.

[5] E. A. Belati and G. R. M. da Costa, "Transmission loss allocation based on optimal power flow and sensitivity analysis," International Journal of Electrical Power \& Energy Systems, vol. 30, pp. 291-295, 5// 2008.

[6] E. A. Belati, C. F. Nascimento, A. B. Dietrich, and H. de Faria, "Sensitivity analysis applied to nodal technical losses evaluation in power transmission systems," International Transactions on Electrical Energy Systems, pp. n/a-n/a, 2012.

[7] C. S. Cheng and D. Shirmohammadi, "A three-phase power flow method for real-time distribution system analysis," Power Systems, IEEE Transactions on, vol. 10, pp. 671-679, 1995.

[8] E. U. E. I. A. (EIA). (2011, 02/11). International Energy Outlook 2011.

[9] V. C. Gungor, D. Sahin, T. Kocak, S. Ergut, C. Buccella, C. Cecati, et al., "Smart Grid Technologies: Communication Technologies and Standards," Industrial Informatics, IEEE Transactions on, vol. 7, pp. 529-539, 2011.

[10] W. T. Huang and K. C. Yao, "New network sensitivity-based approach for real-time complex power flow calculation," Generation, Transmission \& Distribution, IET, vol. 6, pp. 109-120, 2012.

[11] A. Kishore and E. F. Hill, "Static Optimization of Reactive Power Sources by use of Sensitivity Parameters," Power Apparatus and Systems, IEEE Transactions on, vol. PAS-90, pp. 11661173, 1971.

[12] A. Monticelli, Fluxo de Carga em Redes de Energia Elétrica. São Paulo: Edgard Blucher, 1983.

[13] J. Peschon, D. S. Piercy, W. F. Tinney, and O. J. Tveit, "Sensitivity in Power Systems," Power Apparatus and Systems, IEEE Transactions on, vol. PAS-87, pp. 1687-1696, 1968.

[14] W. F. Tinney and C. E. Hart, "Power Flow Solution by Newton's Method," Power Apparatus and Systems, IEEE Transactions on, vol. PAS-86, pp. 1449-1460, 1967.

[15] L. Yan, L. Yulei, Z. Buhan, and M. Chengxiong, "A Modified Newton-Raphson Power Flow Method Considering Wind Power," in Power and Energy Engineering Conference (APPEEC), 2011 Asia-Pacific, 2011, pp. 1-5.

[16] University of Washington - Department of Electrical Engineering. http://www.ee.washington.edu/research/pstca 